\title{
Mitogen-activated protein kinase pathways and cardiac surgery
}

\author{
Tanveer A. Khan, MD \\ Cesario Bianchi, MD, PhD \\ Marc Ruel, MD, MPH \\ Pierre Voisine, MD \\ Frank W. Sellke, MD
}

A list of 74 references is available online.
From the Division of Cardiothoracic Surgery, Beth Israel Deaconess Medical Center and Harvard Medical School, Boston, Mass.

Funding was provided by a grant from the National Institutes of Health, NIH R01 HL46716. Dr Khan is supported by an Individual National Research Service Award from the National Institutes of Health, NIH NRSA 1F32 HL69651.

Received for publication Jan 22, 2003; revisions requested $\mathrm{Feb}$ 10, 2003; revisions received March 4, 2003; accepted for publication April 21, 2003.

Address for reprints: Frank W. Sellke, MD, Chief, Division of Cardiothoracic Surgery, Beth Israel Deaconess Medical Center, 110 Francis St, Suite 2A, Boston, MA 02215 (E-mail: fsellke@caregroup.harvard.edu).

J Thorac Cardiovasc Surg 2004;127:806-11 $0022-5223 / \$ 30.00$

Copyright () 2004 by The American Association for Thoracic Surgery

doi:10.1016/j.jtcvs.2003.04.001
Mitogen-activated protein kinases are serine-threonine protein kinases that are involved in several processes important to cardiac surgery such as vascular permeability, cytokine production, vasomotor function, and reperfusion injury. Mitogen-activated protein kinases are expressed in multiple cell types including cardiomyocytes, vascular endothelial cells, and vascular smooth muscle cells. Mitogen-activated protein kinases function in cellular signal transduction cascades and are activated by a diverse range of stimuli including ischemia, shear stress, and vasoactive agents. Three major mitogen-activated protein kinase families were identified as the extracellular signal-regulated kinases, c-Jun $\mathrm{NH}_{2}$-terminal protein kinases, and p38 kinases. Extensive investigation has established roles for extracellular signal-regulated kinases, c-Jun $\mathrm{NH}_{2}$-terminal protein kinases, and p38 kinases in cardiovascular signal transduction pathways. Activity of these signal cascades may contribute to the increased pulmonary vascular permeability and myocardial reperfusion injury observed after cardiac surgery with cardioplegia and cardiopulmonary bypass. Recent findings from our laboratory suggest that alterations in the activity of myocardial extracellular signal-regulated kinase pathways occur as a result of cardioplegia-cardiopulmonary bypass in humans. In addition, these differences in extracellular signal-regulated kinase activity were shown to mediate coronary microcirculatory dysfunction associated with cardioplegia-cardiopulmonary bypass. The resulting deficit in coronary microcirculatory regulation may potentially lead to detrimental effects on organ perfusion and function. As mitogen-activated protein kinase pathways are further characterized, our potential to develop methods to prevent morbidity associated with cardiac surgery and cardiopulmonary bypass may be greatly improved.

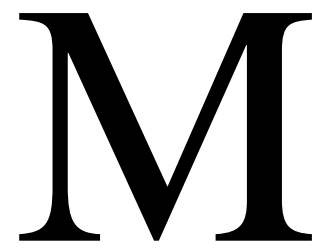

itogen-activated protein kinases (MAPK) are serine-threonine protein kinases that are involved in several processes important to cardiac surgery such as vascular permeability, cytokine production, vasomotor function, and reperfusion injury. MAPK are expressed in multiple cell types including cardiomyocytes, vascular endothelial cells, and vascular smooth muscle cells. MAPK function in cellular signal transduction cascades and are activated by a diverse range of stimuli including ischemia, shear stress, and vasoactive agents. The basic structure of the pathway consists of a sequential, 3-kinase module: a MAPK kinase kinase (MAPKKK) that phosphorylates and activates a MAPK kinase (MAPKK), which in turn activates a MAPK. Three major MAPK families were identified as the extracellular signal-regulated kinases (ERK), c-Jun $\mathrm{NH}_{2}$-terminal protein kinases (JNK), and p38 kinases. Extensive investigation has established roles for ERK, JNK, and p38 kinase in the regulation of cardiovascular signal transduction pathways. Recent findings from our laboratory suggest that the ERK pathway mediates coronary microcirculatory dysfunction associated with cardioplegia and cardiopulmonary bypass $(\mathrm{CPB})$ in humans. In this review, the 


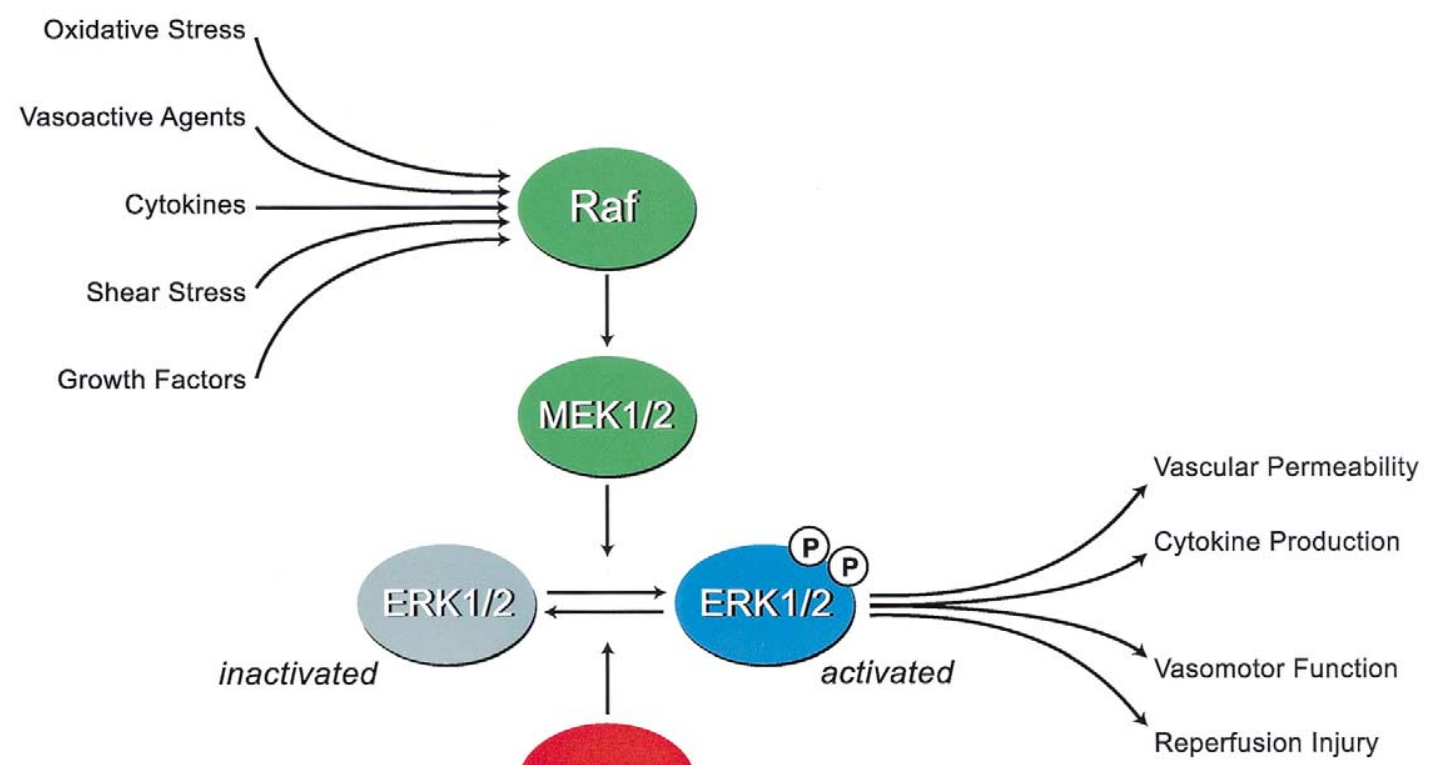

MKP-1

Figure 1. MAPK activation, regulation, and downstream effects. The ERK three-kinase regulatory module is shown as a representative MAPK pathway. Multiple extracellular signals activate the ERK1/2 cascade including oxidative stress, vasoactive agents, cytokines, shear stress, and growth factors. Raf, an MAPK kinase kinase, and MEK1/2, an MAPK kinase, transmit signals to ERK1/2, which is activated by dual phosphorylation. Activated ERK1/2 is involved in several physiologic processes of cardiovascular significance such as vascular permeability, cytokine production, vasomotor function, and reperfusion injury. ERK1/2 is dephosphorylated and deactivated by MKP-1. ERK1/2, extracellular signal-regulated kinase 1/2; MAPK, mitogen-activated protein kinase; MEK1/2, MAPK/ERK kinase 1/2; MKP-1, MAPK phosphatase-1.

characteristics of MAPK signaling pathways will be presented as well as evidence to support MAPK cascades in the regulation of vascular endothelial cell permeability, production of cytokines, modulation of vasomotor function, and mediation of reperfusion injury. In addition, the potential roles of these protein kinases in microcirculatory dysfunction due to cardioplegia and CPB shall be discussed.

\section{MAPK Signal Transduction Cascades}

The three-kinase regulatory module of the MAPK pathways was defined with the identification of the protein kinases upstream of ERK. ${ }^{1}$ MAPK are activated by phosphorylation to create an active, dual-phosphorylated form. The module is a regulatory scheme of sequential protein kinase phosphorylation and activation. MAPK then function to phosphorylate serine and threonine residues of their substrates, many of which are transcription factors, which are accessed after MAPK translocation across the nuclear membrane. ${ }^{2}$ Protein kinase phosphatases such as MAPK phosphatase-1 (MKP-1) also regulate MAPK activity by dephosphorylation, resulting in the deactivation of MAPK. MKP-1 expression is induced by factors such as oxidative stress that also activate the MAPK, thus suggesting a negative feedback loop. ${ }^{3}$

\section{ERK Pathway}

The original MAPK was described as a $42-\mathrm{kDa}$ serinethreonine protein kinase activated by several extracellular stimuli. ${ }^{4}$ The MAPK was named extracellular signal-regulated kinase (ERK) based on the multiplicity of extracellular signals capable of activating the kinase. ${ }^{5}$ Notable among the variety of extracellular stimuli are growth factors, shear stress, and cytokines. ${ }^{6-8}$ Among the several forms of ERK, the 42-kDa (ERK1) and 44-kDa (ERK2) forms are the most widely studied. The ERK three-kinase module is the best described of the MAPK signaling pathways (Figure 1). The Raf kinases function as MAPKKK of the ERK pathway. MAPK/ERK kinase 1/2 (MEK1/2) follows as the downstream MAPKK, ${ }^{9}$ which activates ERK by phosphorylation of the activation loop of the catalytic domain. ${ }^{10}$ ERK phosphorylates substrates in both cytoplasmic and nuclear cellular compartments. Among proteins phosphorylated in the cytosol are ribosomal S6 kinase p90 and cytosolic phospholipase $\mathrm{A}_{2} \cdot{ }^{11,12}$ Cytosolic phospholipase $\mathrm{A}_{2}$ is involved in the production of arachidonic acid, the rate-limiting step in the synthesis of prostaglandins and leukotrienes. ${ }^{12}$ Activated ERK is translocated to the nucleus and capable of phosphorylating multiple different transcription factors including Elk-1. ${ }^{2}$ 


\section{JNK Pathway}

JNK was identified as a MAPK distinguishable from ERK by phosphorylating the transcription factor c-Jun at the $\mathrm{NH}_{2}$-terminal transactivation domain instead of the $\mathrm{COOH}$ terminal site where it is phosphorylated by ERK. ${ }^{13}$ The JNK cascade is activated by several forms of cell stress such as heat shock, radiation, and oxidation, as well as the binding of ligands to extracellular receptors including $G$ proteincoupled, tyrosine kinase, and cytokine receptors. ${ }^{14}$ Noteworthy among cytokines that activate JNK are the inflammatory mediators tumor necrosis factor (TNF)- $\alpha$ and interleukin (IL)-1. In addition to c-Jun, JNK phosphorylates transcription factors such as $\mathrm{p} 53^{15}$ and Elk- $1,{ }^{2}$ which is a point of convergence of the ERK, JNK, and p38 pathways.

\section{p38 Kinase Pathway}

p38 kinase was identified as a $38-\mathrm{kDa}$ protein kinase with $49 \%$ sequence homology to ERK. ${ }^{16}$ p38 kinases also are activated by cellular stress and certain cytokines, notably IL-1 and TNF- $\alpha .{ }^{17-19}$ Accordingly, JNK and p38 kinase are known as stress-activated protein kinases. Similar to ERK and JNK, p38 activation is accomplished by dual phosphorylation of threonine and tyrosine residues. ${ }^{17}$ p38 phosphorylates transcription factors such as Elk- $1^{20}$ and myocyte enhancer factor $2 .^{21}$

\section{Activation of MAPK in Cardiovascular Tissue Cellular Stress}

During cardiac surgery, myocardial tissue is exposed to stresses that are capable of activating MAPK cascades such as shear stress, stretch, and ischemia. In cultured cardiac myocytes, shear stress and mechanical stretch activate MAPK. ${ }^{22}$ Pulsatile stretch also was demonstrated to induce phosphorylation of ERK, JNK, and p38 kinase. ${ }^{23}$ Oxidative stress was shown to activate MAPK, as demonstrated by hydrogen peroxide activating MAPK in both cultured cardiac myocytes ${ }^{24,25}$ and perfused heart models. ${ }^{26}$ Thus, the production of reactive oxygen and nitrogen intermediates during CPB and reperfusion may alter MAPK activity and potentially contribute to processes such as reperfusion injury. ${ }^{27,28}$

\section{Vasoactive Agents and G Protein-Coupled Receptors}

MAPK pathways are stimulated by several vasoactive agents, many of which transmit signals by way of G protein-coupled receptors. ${ }^{29}$ Phenylephrine, endothelin-1, and angiotensin II were shown to activate MAPK. In a perfused rat heart model, ERK, JNK, and p38 kinase were shown to be activated by phenylephrine. ${ }^{30}$ Treatment of cardiac myocytes with endothelin-1 activates ERK1/2, JNK, and p38. ${ }^{31,32}$ Angiotensin II increases ERK activity in rat coronary microvascular endothelial cells and both ERK and JNK activity in rat ventricular myocytes. ${ }^{33}$ In an in vivo animal model, intravenous infusion of angiotensin II acti- vated JNK and to a lesser extent ERK. ${ }^{34}$ These mechanisms of activation are significant in light of the frequency with which phenylephrine, angiotensin-converting enzyme (ACE) inhibitors, and angiotensin II receptor antagonists are used in patients having cardiac surgery. The use of these agents may modify MAPK pathway activity and result in alterations in downstream cellular events that regulate such functions as vascular permeability, cytokine production, and vasomotor tone.

Several lines of evidence suggest MAPK activation via G protein-coupled receptors is instrumental in the development of cardiac hypertrophy. ${ }^{29,35}$ However, in a study of cardiac MAPK in humans, no activation was seen in patients with cardiac hypertrophy. ${ }^{36}$ Further investigation will determine a more complete picture of the role of MAPK pathways in the regulation of the cardiac hypertrophic response.

\section{MAPK in Cardiovascular Physiology Vascular Permeability}

MAPK pathways have been implicated in the development of vascular endothelial permeability, which may become clinically manifest as organ edema and dysfunction. ERK1/2 and p38 kinase were shown to regulate permeability of endothelial cells, ${ }^{37}$ and an in vivo model demonstrated reduced peripheral edema in animals treated with a MEK inhibitor. ${ }^{38}$ Studies using bovine pulmonary artery endothelial cells have demonstrated that regulation of permeability is a function of MAPK pathway activation. ${ }^{39}$ In the isolated, blood-perfused rat lung, ERK1/2 activation was associated with alterations of the capillary barrier. ${ }^{40}$ Finally, p38 was shown to increase endothelial cell layer permeability through alterations in vascular endothelial (VE)-cadherins, components of cellular adherens junctions. ${ }^{41}$ A study from our laboratory demonstrated degradation of VE-cadherins during $\mathrm{CPB}$, providing a potential mechanism of increased vascular permeability. ${ }^{42}$ Thus, MAPK cascades may be involved in mechanisms causing increased vascular permeability during and after $\mathrm{CPB}$, leading to organ edema with potential detrimental outcomes such as myocardial dysfunction and pulmonary insufficiency.

\section{Cytokine Production}

The generation of inflammatory cytokines has been shown to involve MAPK pathways. In an isolated, perfused rat heart model, inhibition of p38 kinase resulted in diminished myocardial TNF- $\alpha$ production after oxidant stress. ${ }^{43}$ p38 kinase also was suggested to play a prominent role in the regulation of IL-1 production by macrophages. ${ }^{44}$ In cardiac myocytes, MAPK were shown to regulate IL-6 gene expression. ${ }^{45}$ In cardiac fibroblasts, IL-6 gene expression induced by angiotensin II was shown to be mediated by ERK and p38 kinase. ${ }^{46}$ A study of human pulmonary vascular endo- 
thelial cells revealed that IL-8 production induced by exposure to TNF- $\alpha$ and IL- 1 is dependent on p38 kinase. ${ }^{47}$ These findings illustrate that in multiple cell types, MAPK mediate cytokine production, which is a prominent feature of the inflammatory response to CPB.

\section{Vasomotor Function}

The ubiquitous nature of MAPK is well illustrated by their role in vasomotor regulation in the cerebral, coronary, pulmonary, gastrointestinal, and renal circulations. MAPK pathways were implicated in cerebral artery contraction induced by low extracellular magnesium concentration. ${ }^{48}$ Similarly, coronary smooth muscle contraction by endothelin-1 was shown to involve a MAPK-dependent mechanism. ${ }^{49}$ p38 kinase and hsp27 were shown to mediate pulmonary vascular smooth muscle contraction. ${ }^{50}$ In mesenteric vessels, inhibition of the ERK and p38 kinase pathways reduces the contractile response to norepinephrine. ${ }^{51}$ Finally, blockade of MAPK activation attenuates renal artery contraction in response to serotonin receptor stimulation. $^{52}$

\section{Reperfusion Injury}

Reperfusion injury constitutes a significant pathophysiologic mechanism in cardiac surgery that contributes to postoperative myocardial dysfunction. ERK, JNK, and p38 kinase were shown to be activated in animal models of myocardial reperfusion injury. ${ }^{53,54}$ In an isolated, perfused heart model of reperfusion injury, ERK1/2 activity was observed to improve cardiac functional recovery, where as p38 kinase activity was related to myocardial dysfunction. ${ }^{55}$ Additional support for a cytoprotective effect of ERK1/2 was shown in a study using the cytokine cardiotrophin- 1 . Treatment of cultured cardiac myocytes with cardiotrophin-1 reduced apoptosis after ischemia and reperfusion by an ERK1/2-dependent mechanism. ${ }^{56}$ Consistent with the proposed role of $\mathrm{p} 38 \alpha$ in apoptosis, ${ }^{57}$ evidence suggests the alpha isoform contributes to reperfusion injury. ${ }^{58}$ Furthermore, p38 inhibition exerts a protective effect from reperfusion injury by prevention of upregulation of adhesion molecules P-selectin and intercellular adhesion molecule-1 and polymorphonuclear leukocyte tissue infiltration. ${ }^{59} \mathrm{Fi}-$ nally, cardiac arrhythmias are well-described manifestations of reperfusion injury, and MAPK were suggested to be involved in the molecular mechanism of atrial fibrillation. ${ }^{60,61}$ Further investigation of the MAPK pathways will lead to a better understanding of the possible protective role of ERK1/2 and proposed detrimental effect of p38 kinase in reperfusion injury that contributes to myocardial dysfunction, seen clinically as postoperative low cardiac output syndrome.

Studies of myocardial ischemia and reperfusion have suggested a role for apoptosis in reperfusion injury. ${ }^{62} \mathrm{Ap}-$ optosis is a specific form of cell death distinct from necrosis and marked by a specific morphology and relative lack of inflammation. ${ }^{63}$ ERK was demonstrated to provide a cytoprotective effect and prevent apoptosis. ${ }^{55,64}$ Despite evidence to support JNK and p38 mechanisms in cardiomyocyte apoptosis $^{65,66}$ other reports document JNK and p38 activity promoting cell survival. ${ }^{67,68}$ Whether JNK and p38 mediate apoptosis or promote cell survival may be influenced by factors such as the particular isoform activated, as seen with $\mathrm{p} 38 \alpha$ and $\mathrm{p} 38 \beta^{57}$ and JNK1 and JNK2, ${ }^{69}$ or the duration of MAPK activation. ${ }^{70}$

\section{Response of MAPK to Cardioplegia and CPB}

Evidence from recent studies suggests that cardioplegia and $\mathrm{CPB}$ cause alterations in the activity of MAPK pathways, potentially resulting in pathophysiologic responses such as coronary microvascular dysfunction. The activity of MAPK pathways in response to $\mathrm{CPB}$ was characterized in tissue from animal models. In a study from our laboratory using a pig model of $\mathrm{CPB}$, elements of the ERK pathway including activated MEK1/2 and activated ERK1/2 were shown to be decreased following CPB in atrial and ventricular myocardial tissue. The activated forms of ERK1/2 were decreased post-CPB, but the total protein levels of ERK1/2 were unchanged. Although levels of activated MEK1/2 and ERK1/2 decreased, MKP-1 levels increased post-CPB. MKP-1, which may be activated by oxidative stress during reperfusion, potentially dephosphorylates and deactivates ERK1/2 post-CPB. ${ }^{71}$ Further investigation of the response of the ERK1/2 pathway to CPB in our pig model revealed that activation of ERK1/2 occurred during CPB, which was then followed by the previously observed deactivation of ERK1/2 during post-CPB reperfusion. Thus, the activity of myocardial ERK1/2 pathways in response to CPB in our animal model is characterized by oscillations seen as activation during $\mathrm{CPB}$ and deactivation to below baseline levels during post-CPB reperfusion. Oscillations in ERK1/2 and MEK1/2 activity were also observed in skeletal muscle and mesenteric vessels, suggesting systemic changes in MAPK activity with potential effects on vascular permeability, cytokine production, and vasomotor function, as well as a contributing role in reperfusion injury. ${ }^{72}$

Recently, we observed that activated ERK1/2 similarly was decreased in atrial myocardial tissue following cardioplegia and CPB in patients during cardiac surgery (Figure 2 ). Consistent with our animal model, MKP-1 levels also were increased post-CPB. In addition, the basal vasomotor tone of coronary arterioles from these patients was significantly reduced post-CPB. Treatment of the human coronary microvessels with a MEK1/2 inhibitor, PD98059, caused a similar loss of basal tone (unpublished data). These findings provided evidence to suggest that decreased ERK1/2 activity post-CPB in part mediates coronary microvascular dys- 

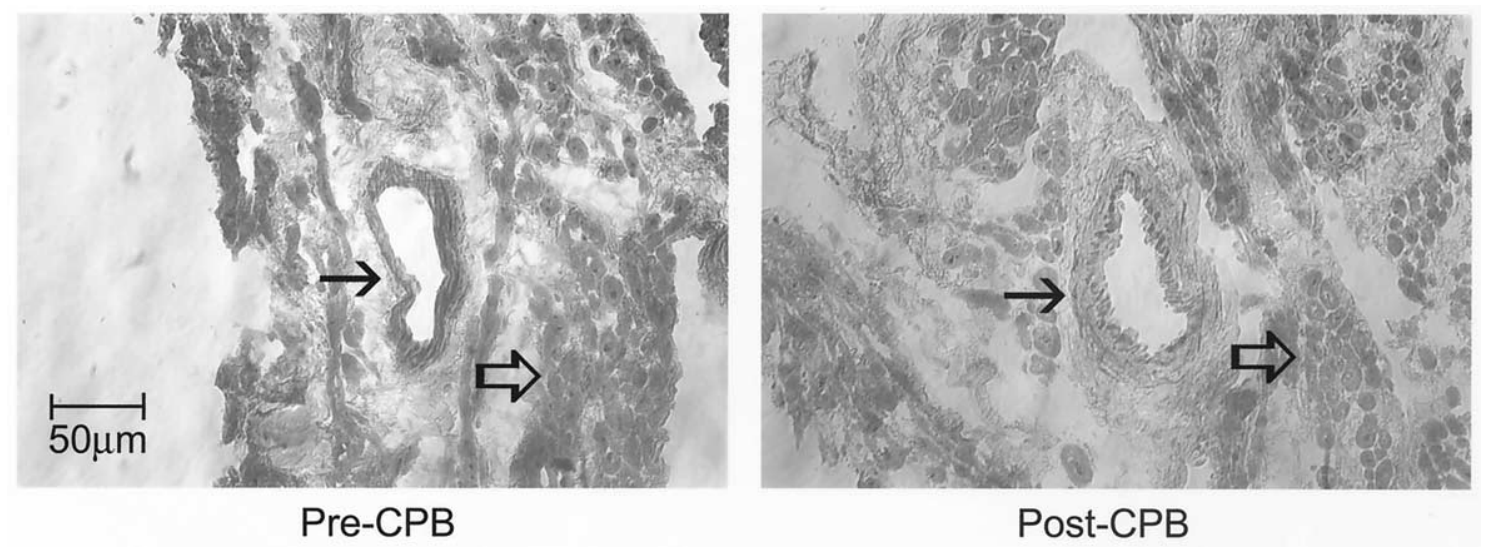

Figure 2. Decreased activated ERK1/2 in myocardial tissue after cardioplegia-cardiopulmonary bypass (CPB). Representative images of immunohistochemical staining showing activated ERK1/2 in coronary arterioles (solid arrows) and cardiomyocytes (open arrows) in human myocardial sections. Staining for activated ERK1/2 is decreased post-CPB (right) compared with pre-CPB (left).

function in patients who undergo cardiac surgery. Dysfunction of the coronary microcirculation due to CPB may result in detrimental changes in myocardial perfusion and function. ${ }^{73}$ A previous report of MAPK activity in atrial myocardial tissue from patients undergoing cardiac surgery described increased levels of activated ERK1/2, JNK, and p38 following cardioplegia and CPB. ${ }^{74}$ The difference may represent a pattern of oscillation between activation and deactivation of MAPK pathways during CPB and reperfusion, which was suggested in the study from our laboratory. ${ }^{72}$ Overall, these studies illustrate that alterations in MAPK cascades are significant during cardiac surgery and suggest a possible mechanistic role for MAPK in coronary microvascular dysfunction. Considering the extensive involvement of these signal transduction pathways in other organ systems and in processes such as vascular permeability and cytokine production, MAPK likely contribute to other aspects of the inflammatory response to CPB as well. Thus, the MAPK signal transduction pathways provide potential sites of intervention to prevent deleterious effects of cardioplegia and CPB.

\section{Conclusions}

MAPK are essential components of signal transduction pathways implicated in several physiologic responses to cardiac surgery. During cardiac surgery, the myocardium is exposed to ischemia, vasoactive agents, and physical stress such as shear force and stretch, all of which alter the activity of MAPK pathways. The increased vascular permeability, vasomotor dysfunction, and cytokine production associated with the inflammatory response to CPB may be mediated in part by MAPK. Significant organ dysfunction may result from derangements in these physiologic processes, which were suggested to involve MAPK pathways in cardiac, pulmonary, renal, mesenteric, and central nervous systems. Activation of the MAPK cascades during reperfusion also may be associated with reperfusion injury as well as cardiac arrhythmias. Finally, we have shown that changes in MAPK activity in patients due to cardioplegia and CPB are involved in coronary microcirculatory dysfunction, with potential detrimental effects on myocardial perfusion. As the mechanisms of MAPK pathways in cardiovascular physiology are further uncovered, our potential to develop methods to prevent morbidity associated with cardiac surgery with cardioplegia and CPB may be greatly improved.

\section{Selected References \\ See online version for complete reference list.}

Aikawa R, Komuro I, Yamazaki T, et al. Oxidative stress activates extracellular signal-regulated kinases through Src and Ras in cultured cardiac myocytes of neonatal rats. J Clin Invest. 1997;100:1813-21.

Araujo EG, Bianchi C, Faro R, Sellke FW. Oscillation in the activities of MEK/ERK1/2 during cardiopulmonary bypass in pigs. Surgery. 2001;130: 182-91.

Araujo EG, Bianchi C, Sato K, Faro R, Li XA, Sellke FW. Inactivation of the MEK/ERK pathway in the myocardium during cardiopulmonary bypass. J Thorac Cardiovasc Surg. 2001;121:773-81.

Bianchi C, Araujo EG, Sato K, Sellke FW. Biochemical and structural evidence for pig myocardium adherens junction disruption by cardiopulmonary bypass. Circulation. 2001;104:I-319-24.

Bogoyevitch MA, Gillespie-Brown J, Ketterman AJ, et al. Stimulation of the stress-activated mitogen-activated protein kinase subfamilies in perfused heart. p38/RK mitogen-activated protein kinases and c-Jun N-terminal kinases are activated by ischemia/reperfusion. Circ Res. 1996;79:16273 .

Boulton TG, Nye SH, Robbins DJ, et al. ERKs: a family of protein-serine/ threonine kinases that are activated and tyrosine phosphorylated in response to insulin and NGF. Cell. 1991;65:663-75.

Boulton TG, Yancopoulos GD, Gregory JS, et al. An insulin-stimulated protein kinase similar to yeast kinases involved in cell cycle control. Science. 1990;249:64-7.

Cain AE, Tanner DM, Khalil RA. Endothelin-1-induced enhancement of coronary smooth muscle contraction via MAPK-dependent and MAPK- 
independent $[\mathrm{Ca}(2+)](\mathrm{i})$ sensitization pathways. Hypertension. 2002;39: 543-9.

Clerk A, Fuller SJ, Michael A, Sugden PH. Stimulation of "stress-regulated" mitogen-activated protein kinases (stress-activated protein kinases/ c-Jun N-terminal kinases and p38-mitogen-activated protein kinases) in perfused rat hearts by oxidative and other stresses. J Biol Chem. 1998;273: 7228-34.

Clerk A, Sugden PH. Activation of protein kinase cascades in the heart by hypertrophic G protein-coupled receptor agonists. Am J Cardiol. 1999;83: 64H-69H.

Crews CM, Erikson RL. Extracellular signals and reversible protein phosphorylation: what to Mek of it all. Cell. 1993;74:215-7.

Han J, Lee JD, Bibbs L, Ulevitch RJ. A MAP kinase targeted by endotoxin and hyperosmolarity in mammalian cells. Science. 1994;265:808-11.

Hsu HY, Wen MH. Lipopolysaccharide-mediated reactive oxygen species and signal transduction in the regulation of interleukin-1 gene expression. J Biol Chem. 2002;277:22131-9.

Meldrum DR, Dinarello CA, Cleveland JC Jr, et al. Hydrogen peroxide induces tumor necrosis factor alpha-mediated cardiac injury by a P38 mitogen-activated protein kinase-dependent mechanism. Surgery. 1998; 124:291-6 (discussion 97).

Nwariaku FE, Chang J, Zhu X, et al. The role of p38 map kinase in tumor necrosis factor-induced redistribution of vascular endothelial cadherin and increased endothelial permeability. Shock. 2002;18:82-5.

Ohanian J, Cunliffe P, Ceppi E, Alder A, Heerkens E, Ohanian V. Activation of $\mathrm{p} 38$ mitogen-activated protein kinases by endothelin and noradrenaline in small arteries, regulation by calcium influx and tyrosine kinases, and their role in contraction. Arterioscler Thromb Vasc Biol. 2001;21:1921-7.

Ping P, Zhang J, Cao X, et al. PKC-dependent activation of p44/p42 MAPKs during myocardial ischemia-reperfusion in conscious rabbits. Am J Physiol. 1999;276:H1468-81.

Pulverer BJ, Kyriakis JM, Avruch J, Nikolakaki E, Woodgett JR. Phosphorylation of c-jun mediated by MAP kinases. Nature. 1991;353: 670-4.

Ragette R, Fu C, Bhattacharya J. Barrier effects of hyperosmolar signaling in microvascular endothelium of rat lung. J Clin Invest. 1997;100:685-92. Rossomando AJ, Payne DM, Weber MJ, Sturgill TW. Evidence that pp42, a major tyrosine kinase target protein, is a mitogen-activated serine/threonine protein kinase. Proc Natl Acad Sci U S A. 1989;86:6940-3.

Sellke FW, Boyle EM Jr, Verrier ED. Endothelial cell injury in cardiovascular surgery: the pathophysiology of vasomotor dysfunction. Ann Thorac Surg. 1996;62:1222-8.

Talmor D, Applebaum A, Rudich A, Shapira Y, Tirosh A. Activation of mitogen-activated protein kinases in human heart during cardiopulmonary bypass. Circ Res. 2000;86:1004-7.

Taniguchi T. Cytokine signaling through nonreceptor protein tyrosine kinases. Science. 1995;268:251-5.

Whitmarsh AJ, Shore P, Sharrocks AD, Davis RJ. Integration of MAP kinase signal transduction pathways at the serum response element. Science. 1995;269:403-7.

Yue TL, Wang C, Gu JL, et al. Inhibition of extracellular signal-regulated kinase enhances ischemia/reoxygenation-induced apoptosis in cultured cardiac myocytes and exaggerates reperfusion injury in isolated perfused heart. Circ Res. 2000;86:692-9. 